\title{
From Standardization to Adaptation: Clinical Trials and the Moral Economy of Anticipation
}

\author{
Catherine M. Montgomery
}

Amsterdam Institute for Social Science Research, University of Amsterdam, The

Netherlands

\begin{abstract}
Hailed as the gold standard, the Randomized Controlled Trial (RCT) occupies a hegemonic position at the top of Evidence Based Medicine's hierarchy of knowledge. It is testament to the methodology's capacity for standardization that it can so readily be spoken of in the singular: the RCT. Under what conditions, then, is it possible to speak of change in the gold standard? Since the 1950s, alternative versions of the RCT have been advocated for under the banner of 'adaptive design'. Adaptive designs allow investigators to make pre-planned changes to a trial on the basis of accruing information while the experiment is ongoing. Initially a niche topic of methodological debate among biostatisticians, the approach is becoming widespread in mainstream drug development. A genealogical analysis exposes the discursive moves used to justify and popularize adaptation, from a focus on patient wellbeing and the greater good in the 1960s and '70s, to efficiency and virtualism in the 1990s and 2000s. Changing discourses of time and patienthood have facilitated a move away from standardization as the singular logic of trials towards an appreciation of flexibility, undergirded by probabilistic methodologies. Adams et al's (2009) conceptual framework of anticipation illuminates this evolving moral economy of medical research, in which modes of knowledge production which claim to know the future are supplanting the traditional certainties of fixed and standardized experimental designs. Predictable uncertainty is the currency of this emerging economy, which capitalizes on computer simulation and ever more sophisticated tools of prediction to leverage credibility.
\end{abstract}

Keywords: Clinical trials, adaptive design, time, patients, statistics, anticipation 


\section{Introduction}

Lang talked fast. She seemed to do everything at maximum speed, aware of the extreme urgency of the situation. "It usually takes a year to 18 months to set up a study, and usually epidemics have come and gone in 18 months, so there are never trials within an epidemic," said Lang. "But what we've been able to do with Ebola...we're doing everything in parallel. We're still writing the protocol, we're filling out the ethics application forms, we're sorting out the drug supply, we're sorting out the sites all in one line.” (Bosely 2015)

The West African Ebola epidemic that erupted in 2014 sparked many debates about how best to deploy science to save lives in the context of emergency. One aspect of this was how to quickly test treatments and vaccines so that robust evidence could be obtained while exposing the fewest people possible - both immediately and in the future - to this devastating disease. Controversy raged between North American and European groups over various design features, including whether or not to randomize patients to a standard of care arm, given a case fatality rate of up to $70 \%$ (Adebamowo, Bah-Sow et al. 2014, Cox, Borio et al. 2014, Joffe 2014, Kanters, Thorlund et al. 2015). Lines were drawn between those who insisted it was scientifically imperative to have an experimental control group, and those who believed it was ethically indefensible not to offer all patients a potentially beneficial treatment in the absence of an effective therapy. In spite of these differences, the use of adaptive designs was reportedly adopted by both sides as the methodology of choice (Hayden 2014). For example, in the UK, the Wellcome Trust funded trials using an adaptive multistage approach (Whitehead, Olliaro et al. 2016), while in the US, scientists funded by the Gates Foundation proposed a Bayesian adaptive trial using response adaptive randomization (Berry, Petzold et al. 2016) ${ }^{i}$. Where once adaptive designs were a niche topic of theoretical debate in biostatistics journals, they were now making the mainstream press. How did this change come about, and what is its broader significance?

'Adaptive design' is a loose designation that refers to clinical trials which make use of data accumulating during the study in order to make prospective changes to the design. This could be in relation to the number of treatment arms, the randomization schedule, or even outcome measures. Lang, the scientist at the centre of The Guardian newspaper report cited above, has been a keen advocate of adaptive designs in the field of Global Health. According to her, 'one of the main features is that this approach moves away from the standard phase I, II, and III model of separate protocols to a single study that adapts flexibly in real time as knowledge is gained' (Lang 2011: 967). The real-time flexibility to which Lang refers comes by virtue of a design approach in which potential changes are anticipated and pre-specified in the protocol, and decisions made on the basis of early 'looks' at the data. These kinds of interim analyses can also occur in conventional trials, but are not conducted frequently and do not lead to design changes, which must be declared in advance in order to maintain the statistical validity of the experiment.

What is radical about adaptive designs is their break with the traditional view that the randomized controlled trial (RCT) should garner evidence of an intervention's effect notwithstanding the social contingencies of its implementation. This has meant that traditionally, a trial protocol is designed and approved before the trial starts, the trial 
then runs according to said protocol, and at the end, the data is analysed and an answer to the original question is produced. Changes are not made on the basis of accumulating data, and once the trial starts, its course is set. Adaptive designs, by contrast, are based on the idea that learning can and should take place during the life of the trial and that it is possible to maintain the trial's integrity if potential fixes are built in at the design stage.

Adaptive designs were first mooted in the 1950s and it has taken decades for their popularity to rise. Today, advocates commonly justify them as an alternative to conventional RCTs on the basis that they are efficient and accelerate the drug testing process. This has not always been the case. Since the 1950s and '60s, the rationale for adaptation has changed markedly, and along with it, the conception of the patient/trial participant. In this paper, I ask how it is possible for the very notion of adaptation in trial design to become an accepted part of drug testing when what has for so long been regarded as the gold standard in medicine is based on the opposite notion of a fixed protocol implemented within a highly controlled clinical environment.

How is change at the top of the evidence-based medicine hierarchy possible, and how is adaptation coming to replace standardization as a virtue? This paper maps the changing discourse around adaptation in medical experimentation. In the process, it identifies changes to the way in which the medical experiment is conceived, in particular its temporal trajectory, or what, following Adams et al, we might refer to as its 'politics of temporality' (Adams, Murphy et al. 2009: 246).

The paper proceeds by charting the changing discourse of adaptation in clinical experimentation chronologically, drawing out three key trends in the process. As I will go on to demonstrate, adaptation has successively (and arguably, successfully) been framed as a means of saving patients, a means of saving the pharmaceutical industry, and a means of saving time itself. We witness this through corresponding references to ethics, efficiency and the virtual, a progression which marks a growing abstraction from individual patient to institution to spatiotemporal dimension. Using examples from the literature, as well as extracts from interviews with key informants, I illustrate how clinical experimentation is moving towards a moral economy of anticipation, where value is located in the probabilistic rather than in the promissory, so long the sign under which trials have been said to reside (Sunder Rajan 2007). In the concluding section, I discuss the practical and theoretical implications of this move, including the situatedness of evidence in medicine and the regulation of clinical research.

\section{Analytic Perspectives}

There has been a long theoretical engagement with clinical trials on the part of anthropologists, sociologists and science studies scholars, at both micro and macro levels. At the micro level, a great deal of this has turned on a critique of standardization and the idea that experiments can be conducted in a way that divorces them from their social context. Focusing on clinical work in relation to guidelines and protocols, this literature characterizes standardization as an ongoing process of situating and adjusting, work which requires constant negotiation and buy-in from 
multiple stakeholders (Berg 1997, Timmermans and Berg 1997, Timmermanns and Berg 2003). At the macro level, the political economy of clinical trials has led to sharp critiques of the neoliberalisation of drug development and the enrolment of vulnerable populations into potentially exploitative regimes of commercial experimentation (Sunder Rajan 2007, Fisher 2009, Petryna 2009).

Both of these perspectives provide valuable theoretical lenses for understanding changes to the way knowledge is produced in evidence based medicine. In this paper, however, my concern is with a historical excavation of the present (Armstrong 2002), based on texts and accounts from key informants. Time, patienthood, and value are key themes in these sources, and it is therefore to the analytic antecedents in the political economy of trials that I turn below.

\section{Political Economy of the Promissory}

The story of adaptive design is one of ethics and efficiency, of the patient and of time. Numerous anthropological and sociological studies have demonstrated how patients have been regarded as a scarce resource in clinical research, with trialists going to ever greater lengths to source new patient populations (Sunder Rajan 2006, Sunder Rajan 2007, Fisher 2009, Petryna 2009). A trend within this literature has been to focus on the spatial expansion of the clinical trials industry and its so-called offshoring, that is, outsourcing clinical trials to areas of the world where experimental labour is cheap and plentiful. Far less has been written about time or indeed about time as a scarce resource, even though the analysis of the pharmaceutical industry as a failing endeavour repeatedly references the lengthy timelines of drug discovery and testing as one of its greatest maladies. When time is invoked, it is usually through the analytic lens of the promissory (e.g. Davis and Abraham 2013, Lezaun and Montgomery 2014).

Promissory regimes have been closely intertwined analytically with the capitalization of the life sciences. Sociologists and anthropologists have identified the processes characterising this as 'biocapital' (Sunder Rajan 2006), 'biovalue' (Waldby 2000, Waldby 2002) and 'bioeconomics' (Rose 2001), amongst others. The analyses draw on the Marxist language of commodity-based processes, focusing on the notions of capital, value and surplus value. Sunder-Rajan's Biocapital, in particular, makes the link between the implosion of the sciences with capitalism and a new reliance on the promissory. Speculative capitalism in the guise of venture science relies on hype and forward-looking statements (so-called 'promissory conjuration') to create (commercial) value in the present, albeit in the absence of tangible events, products or outcomes. Genome science is a key exemplar of this phenomenon, with many millions of dollars invested in large-scale projects, such as the Human Genome Project, on the basis of great scientific promise, but few, if any, tangible products.

The promissory is a means of 'calling on the future to account for the present' by way of projecting visions of how things must be (Sunder Rajan 2006: 116). To this extent, it is a potentially useful way of thinking about adaptive designs analytically. However, while promissory regimes project confidence in the face of uncertainty, this is not the goal of adaptive designs, which rather use a more complex set of techniques to negotiate the unknown, present alternative scenarios and prepare for ways to 
change ongoing experiments in response to empirical data. Furthermore, while Sunder-Rajan and others articulate the role of promissory conjuration in relation to the commercialization of US biotech, the temporal politics of adaptive design straddle a far more diffuse set of scientific institutions, from profit-seeking big Pharma to publicly- and philanthropically-funded academia. What is at stake is not so much the creation of commercial value through the promise of a given vision, but the creation of value - moral, epistemic and commercial - through the ability to know the unknowns and to fix the future as it unfolds.

\section{Anticipatory Regimes}

I therefore turn to Adams et al's (2009) work on anticipation to analyse how adaptation has become an acceptable part of clinical experimentation. Anticipation references a mode of 'thinking and living toward the future', in which new opportunities are created to reconfigure the possible. Key to this is an abandonment of the actual for modes of knowledge production which claim to know the future, such as prediction, speculative forecast and computer simulation. Certainty is no longer the currency of this moral economy, but rather predictable uncertainty, which both fosters preparedness and incites perpetual striving for new ways to know the future.

Adams et al disassemble anticipation into a number of key dimensions: injunction, abduction, optimization, preparedness, and possibility. Injunction signifies the moral valence of anticipation, 'an obligation to "stay informed" about possible futures' that goes beyond the merely epistemic and that heralds 'the emergent "almost" as an ethicized state of being' (Adams, Murphy et al. 2009: 254). Abduction refers to a mode of acting in the face of contingency and uncertainty, premised upon moving back and forth between empirical information and abstract ways of thinking. 'Abduction moves reasoning temporally from data gathered about the past to simulations or probabilistic anticipations of the future that in turn demand action in the present... the openness, fluctuations and instabilities of abduction as lived experiencing of "trying to know" what to do in time, what action to take "before" in preparation for the inevitable yet avoidable "after," are central' (Ibid: 255). Some key methods of abduction are computer modeling and simulation, which, as will become apparent later in the paper, are central techniques in adaptively designed trials.

Adams et al use optimization to characterize the way in which anticipation impels actors to maximize their chances for the best possible future, and the way in which the scope of this is limitless. This is because anticipation does not rely on actual events, but on imagined possibilities. Preparedness refers to speculative and reactive actions which treat future events as if they were already here. Finally, possibility: anticipatory regimes create demand for novel technologies, economies and politics in order to satisfy preparedness and optimization: 'Management of the future becomes a preoccupation of the present through the obligatory passage-point of "possibility." ...It is possible, so the injunction goes, to manage the anticipated.’ (Ibid: 259).

While many of these facets of anticipatory regimes are relevant to the current analysis, of primary interest to this paper is the way in which they affect our 
experience of time and space, bringing the future into the present, and expanding the physical realms of the possible:

Anticipatory regimes, like those of capitalism, tend to work through logics of expansion, in which new territories for speculation must be continually found to keep the anticipatory logic moving. Anticipatory regimes expand their scope of inclusion, elongate their reach in time, in space, and in phenomenological terms. (Adams, Murphy et al. 2009: 250-251)

To paraphrase Adams et al, this results in a spatial and temporal reterritorialization of the domains and sites which are called into the future. In relation to adaptive design, we can already perceive hints of this in Lang's words above, in which she notes that the field of experimentation which was traditionally demarcated by separate trial protocols and discrete questions has now come to be considered a potentially limitless landscape of experimental possibility.

\section{Genealogical analysis}

The role of adaptation in medical experiments has predominantly been the concern of three groups: biostatisticians, medical ethicists and drug regulators. More recently, it has begun to attract the interest of the medical community at large. The analysis in this paper is based primarily on debates within statistics, but also looks at the diffusion of these debates into the mainstream medical and scientific press. To examine these debates, I conducted a genealogical analysis. Genealogy is a means of demonstrating the contingency of what we take for granted by showing apparently natural categories to be historically-contingent constructs. Expounded in the work of Michel Foucault, it has subsequently been used by medical sociologist David Armstrong to analyse the history of developments in medical science, from population screening to patient behavior (Armstrong 2012, Armstrong 2014).

The arguments in this paper are based on an analysis of journal articles, editorials and correspondence in the biostatistical and medical literature. Through a search of the PubMed and Web of Science databases, I identified published material referencing the words 'adaptive' or 'flexible' and 'clinical trial'. A process of filtering out irrelevant papers then took place (e.g. papers on adaptive immunity and flexible sigmoidoscopy), and additional hand searches were made to find references missed by these searches. Articles were analysed for the use and context of the descriptors 'adaptive' and 'flexible' (and their other parts), with particular attention paid to arguments for and against adaptive trial designs.

Alongside the process of analyzing the literature, interviews were conducted with 25 key informants with active methodological and/or practical experience of adaptive design trials. Informants were selected in three ways: firstly, known contacts in the field of trial methodology were approached; secondly, additional contacts were identified through snowballing; and third, key informants were identified through the methodological literature, conferences and workshops. They included fifteen statisticians, five clinicians, two clinical trials project managers and a software developer. The majority worked in academia $(n=20)$, with small numbers representing industry $(n=3)$ and humanitarian non-governmental organizations $(n=2)$. 
Most of the informants $(n=21)$ were located in European institutions in the UK, the Netherlands and Germany, with a minority based in the US $(n=4)$. Interviews took place August 2013 - 2015.

Interviews were transcribed and analysed alongside the literature, using NVivo as a tool for data management and analysis. The latter involved an iterative process of initial line-by-line coding using inductively generated codes, leading to the development of a set of provisional categories. These were then used to code subsequent texts in a more focused manner. Analytic lines of inquiry were explored through memo-writing and constantly turning between the data and the codes to check the adequacy of theoretical categories.

\section{Ethics: Saving the Patient}

In the 1960s, the statistical world was alive with debate about adaptive treatment assignment, a method which uses accumulating information in the trial to randomize the most patients to the best treatment. Key figures in this debate were Anscombe (1963) and Colton (1963), with their loss function models and subsequently Zelen, with his play-the-winner design (Zelen 1969). In the latter, if a patient has a successful outcome on a treatment, a future patient is assigned to the same treatment; if the treatment outcome is a failure, a future patient is assigned to the alternative treatment.

Zelen's play the winner design was radical because rather than the trial using a fixed randomization probability, the probability of a patient being assigned to one treatment or another depended on information which became available as the experiment progressed. Such ideas had already been voiced as early as 1933, when Thompson argued that:

...there can be no objection to the use of data, however meagre, as a guide to action required before more can be collected...If such a discipline were adopted, even though it were not the best possible, it seems apparent that a considerable saving of individuals otherwise sacrificed to the inferior treatment might be effected. This would be important in cases where either the rate of accumulation of data is slow or the individuals treated are valuable, or both. (Thompson 1933: 285)

Foremost to the argument of using accumulating data to make decisions as the experiment progresses is the notion of the 'saving of individuals otherwise sacrificed to the inferior treatment'. Throughout the debates of the 1960s and 1970s, this was a recurrent trope, which formed the bedrock of the justification for adapting trials. Adapting the randomization schedule in the trial, it was argued, was a solution to the ethical problem of how to administer a treatment of unknown efficacy to patients when during the course of the trial, its efficacy one way or the other became increasingly apparent.

'Saving patients' was also a question of the length of the experiment, or what I will refer to as trial time. Zelen suggested that 'the guiding idea behind most of the newer 
techniques is the ethical one of not prolonging a trial longer than necessary, for a trial which is unduly prolonged may result in an excessive number of patients being given the less beneficial treatment' (Zelen 1969: 131). We thus see adaptation framed as a question of intervening in trial time, and time itself as a question of ethics. According to Zelen, new statistical techniques were needed to reduce time to be more ethical. Methodological change was coming about not as the result of a theoretical scientific impulse, but because of an ethical one directly linked to clinical practice (it is not insignificant that Zelen's paper was published in a leading medical journal, rather than a statistics journal).

Making explicit the link between 'saving patients' and trial time allowed the tension between clinical practice and scientific experiment to be voiced and to be addressed through methodological means. A key concept thus became the 'patient horizon', referring to an estimate of the total number of patients either on the trial or affected by the trial results in the future via the choice of the best treatment. The concept came about rhetorically through discussion of the ethical need to balance the sacrifice of current study subjects with the benefits which would accrue to future patients. This sacrifice was couched in terms of treatment uncertainty and drew on a specific temporal trajectory of medicine. Weinstein epitomized the view that clinical uncertainty in the present made trial participation a sacrifice:

....any decision rule for allocating patients to clinical procedures in any way other than according to the best interest of the subject at hand does entail a sacrifice on the part of the subject, even if the sacrifice involves trading one very uncertain lottery for another very uncertain, but slightly less desirable lottery. Such sacrifices are often desirable from the point of view of social welfare and medical progress. One is therefore led to consider the implications of this transfer of welfare from today's subject to tomorrow's patient (Weinstein 1974: 1279).

In Weinstein's terms the patient's sacrifice was one of subjecting him or herself to an 'uncertain lottery'. Uncertainty was portrayed as the enemy, both for patients and clinicians, and it was sacrificing oneself to uncertainty which underpinned the methodologists' and medical ethicists' debates about adaptation (see also Simon 1977, Pocock 1979). Already in these early discussions, practices of anticipation were changing the way the RCT was considered. The moral injunction to anticipate the burdens not only of today's subject but of tomorrow's patients led to theoretical debate in statistics about how to optimize the future in the present.

Weinstein advocated adaptive design, and beyond this, an abandonment of discrete experiments in favour of a continuous updating of the standard of care: 'the optimal strategy amounts to an abandonment of the notion of a fixed experiment in favor of a continuous process of applying therapies and updating probabilities for the various possible outcomes of these therapies' (Weinstein 1974: 1282). This, he argued, would take into consideration the wellbeing of both the present experimental subject and the future patient who would benefit from the experiment. In a move that anticipated developments only just being seen today ${ }^{\mathrm{ii}}$, he argued that:

...the dividing line between "experiment" and "routine" should really not exist at all. In terms of the accumulation of clinical knowledge, an experiment 
should not be thought of as a discrete entity but rather as one phase of the ongoing experience with a clinical procedure...Thus, the "experiment" never really ends... (Weinstein 1974: 1284)

Trial time, under Weinstein's formulation, became continuous not discrete, while the patient/subject remained at the centre of the argument for adaptation. Rather than shortening the duration of any given trial, as was Zelen's objective, Weinstein's proposal reformulated experiment as routine and therefore did away with its temporal circumscription.

In practice, early attempts to intervene in trial time by updating a trial's randomization probabilities were met with widespread criticism. In a series of trials assessing extracorporeal membrane oxygenation (ECMO) in neonatal respiratory failure, adaptive designs were used to try and overcome the ethical dilemmas of equipoise and randomization facing clinicians (Bartlett 2014). While these succeeded both in shortening trial time and in producing a statistically significant result, importantly, they failed to convince clinicians (Soll 1996). The first trial used a randomized play the winner design, in which only one patient was randomized to the control group and eleven to ECMO (Bartlett, Roloff et al. 1985). The patient in the control group died while the patients in the intervention arm all survived, demonstrating a highly significant advantage for ECMO $(\mathrm{P}=0.0000001)$. The second trial used a different play the winner design, in which patients were randomized equally until there were four deaths in one arm. Thereafter, patients were allocated to the other arm until statistical significance was reached. After four deaths in the control arm, a further ten patients were assigned to ECMO, and all survived (O'Rourke, Crone et al. 1989).

Criticism of the trials was intense, both in the scientific and the popular press, with one of the two trials the focus of a piece in the Boston Globe, which publicly accused the researchers of being unethical (Knox 1989). Other scientists likewise queued up to damn the designs, with Elliott, for example, calling the analysis of the adaptive trials 'a sad commentary on the application of scientific standards to clinical investigation' (Elliott 1991: 477). The criticisms were wide-ranging: the unconventional designs were impossible for ordinary clinicians to understand; the numbers of patients enrolled were too small; the investigators' preconceptions and emotions predominated over reason; the randomized informed consent (only obtained from those in the intervention arms) was unethical; too few patients were randomized to the control arm; too many patients were randomized to the control arm; and so on (Lantos and Frader 1990, Miké, Krauss et al. 1993, Malcontent 1997).

Perhaps the most insightful comments that the ECMO trials provoked, however, concerned the irreducible nature of uncertainty in medical research and the folly of striving to defeat this. This formed the basis of the papers by both Lantos and Frader and Miké et al, who to varying degrees urged a more open rejection of the 'pursuit of a chimerical scientific perfection' (Lantos and Frader 1990: 412) and instead, attention to 'the basically ambiguous nature of statistical methodology' (Miké, Krauss et al. 1993: 216). Both underscored how the formal processes of mathematics should not be considered the primary determining factor for decision-making in medicine. The taming of uncertainty as part of an effort to control experimental time thus remained a largely theoretical pursuit for the coming decades. 


\section{Efficiency: Saving Industry}

Adaptive designs, then, did not enjoy a huge amount of popularity in the decades immediately following their methodological elaboration, with the ECMO controversy severely denting the credibility of the concept. In the mid- 2000s, however, after a period of renewed activity in the statistical literature, and the Federal Drug Administration's (FDA) (2004) launch of the Critical Path Initiative, interest started to grow within the pharmaceutical industry. The Critical Path Initiative was designed to modernise drug development and testing and specifically to 'improve predictability and efficiency along the critical path from laboratory concept to commercial product' (FDA 2004). Adaptive designs were subsequently listed as an explicit focus in the FDA's (2006) Critical Path Opportunities List under the topic 'Streamlining Clinical Trials'.

In Spring 2005, the Pharmaceutical Research and Manufacturers of America (PhRMA), a large and influential Washington-based lobbying organization, ${ }^{\text {iii }}$ formed a series of working groups to look into the declining success of drug development research in the pharmaceutical industry (Bornkamp, Bretz et al. 2007). One of these was the PhRMA Working Group on Adaptive Designs. In 2006, the group jointly organized a workshop on the topic with the FDA and published a white paper, with the aim of fostering wider use and regulatory acceptance of the methodology (Gallo and Krams 2006). An influential statistician based in a large pharmaceutical company described developments within industry as follows:

[W]hen company managers hear things like this, you know, it's like 'gee, we can change the sample size, and a study that would have been unsuccessful now becomes successful!' I think there's sometimes a reaction like, 'well, finally those statisticians have come up with something useful'. And I think that was driving a lot of this in this 2005 timeframe and companies wanted to get on board.

Reflecting this, the industry press exploded with articles about adaptive design, going from just two mentions in the period 2000 - 2005 to nearly 1,700 in the period 2005 $2010^{\mathrm{iv}}$. During this period, industry was active in engaging regulators on the subject of adaptive designs, for example sponsoring workshops with the European Medicines Agency (EMA), the FDA and Japan's Pharmaceuticals and Medical Device Agency (Chuang-Stein, Bretz et al. 2009).

The key to this change in attitude towards adaptation was the translation of the concern of ethics into one of efficiency, via the notion of trial time. Whereas early advocates of adaptive design linked time to the individual patient/subject, industry advocates saw the benefits of reduced timeframes for company bottom lines. The discourse moved from saving patients to saving time, and thereby - by implication saving costs and thus the pharmaceutical industry itself.

That the traditional model of drug development has led to an ailing industry is now well described and oft-repeated (for a representative summary, see Woodcock and Woosley 2008: 3). Diagnosing this malady in typical fashion, Holford writes: 
Typically, only 1 out of 1,000 potential drugs reaches the clinical trial stage, with 6 years of research behind it and then another 8 years between entering clinical trials and receiving approval from regulatory agencies for sale to the public. Approximately $90 \%$ of drugs fail during clinical development, and the total cost of bringing a new medication to the market is as high as US\$1.7 billion. (Holford, Ma et al. 2010: 166)

The discourse of efficiency, which advocates of adaptive designs propounded, could not have been a more fitting tonic for industry executives troubled by such statistics. The literature on adaptive designs from the mid-2010s on reflects this concern with efficiency, with ethics now taking a back seat. Adaptive designs are justified theoretically on the basis of greater efficiency, but reports of their implementation also point to this as a tangible outcome in practice. Evincing this, Hutson writes in a 2009 article in Nature Medicine that:

Wyeth alone reports that, over the last two years, it has performed six dosefinding adaptive clinical trials, with which they credit a savings of \$19 million and nine months of development time. (Hutson 2009: 977)

Similarly extolling the virtues of efficiency, Jones (2010) observes that any potential delays in designing adaptive trials at the outset 'can be more than offset by cutting down the time taken to run the trial itself - a year or more in some cases'. Furthermore, integration of trial phases into a single trial 'could lead to more rapid product registration' (Jones 2010: 2-3). Efficiency is clearly conceptualized here as time foreshortened in the experimental process. It is no longer linked to patient wellbeing, as it was in the 1960s and '70s, but instead indexes the wellbeing of the pharmaceutical industry. In this way, big Pharma exploited the joint facets of ethicality and financial potential inherent in anticipatory regimes to re-frame the industry's sense of the possible.

In the move from ethics to efficiency, the patient undergoes a re-signification. In the same paper by Jones, the head of clinical trial optimization at Eli Lilly, a large pharma company, is quoted as saying: 'This method isn’t just about speed, but about greater information value per patient, making better decisions and generating a more informative package when it comes to filing' (Jones 2010). The emphasis here is on 'greater information value per patient'. The patient has become an economic unit, part of a value calculation that is about information rather than medical care. This is a recurrent trope in the published literature, in particular where the goal is drug registration by the FDA.

Coterminous with the discursive transition from saving patients to saving time is the discourse of not wasting patients. This, again, is linked to the trend above of conceptualizing patients as commodity. No longer merely sick individuals, patients are now reservoirs of information and extracting maximum information value per patient becomes the predominant concern. This is exemplified in a paper reporting the design of a specific adaptive trial to evaluate treatment for septic shock (Lewis, Viele et al. 2013). As we might expect, the paper is replete with references to efficiency and inefficiency in adaptive and traditional designs respectively (17 mentions of efficiency in its various forms over the 5 page article). Of greater interest, however, is the re-insertion of ethics into the efficiency equation in the guise 
of the 'ethical balance'. This refers to the balance between patient risk, benefit and acquired information - with acquired information being a notable addition to what is usually simply referred to as the balance between patient risk and benefit. This is in keeping with the reconceptualization of medical science as an information science, with adaptive designs more heavily dependent on data services (and the software this requires) to supply information in real-time as the trial progresses.

The result of inserting 'acquired information' into the risk-benefit equation is that the experimental subject is reconceptualised as a source of data rather than a living, breathing individual; in traditional ('inefficient') designs, 'these subjects could be considered in effect "wasted" as they yield little useful information'. By contrast, in an adaptive trial, such as the one proposed in the paper, 'the trial efficiently allocates subjects to the best dose arm, maximizing the information gained per subject' (Lewis, Viele et al. 2013: 1677). This contrasts sharply with the discourse of the early adaptive design papers, in which it was the clinician - not the disembodied trial who was agentive in allocating patients to one treatment or another. Whereas in Zelen's time, adaptive treatment allocation was proposed as a solution to clinical discomfort with the ethics of randomization, thirty years later, both clinician and patient subjectivities have been supplanted by the de-personalized figures of the trial and trial data.

\section{The Virtual: Saving Time}

Such de-personalization might seem at odds with personalized medicine, often cited as a key beneficiary of progress in adaptive design methodology. One of the flagship projects of adaptive design proponents, the I-SPY trials, uses a Bayesian design to find improved treatments for subsets of patients with breast cancer on the basis of the molecular characteristics of their disease (Barker, Sigman et al. 2009). Extolling the advantages of this approach, the authors write that, "the trial is designed to "learn" over time which profiles predict response to each drug' (Ibid: 99). The basis of this learning is mathematical modeling, which uses information obtained during the course of the trial to continually update the predictive probabilities of future observations (Berry 2006). Replacing the art of a clinician to learn experientially visà-vis their individual patient, the science of medical practice, reconfigured as a decision science, is now delegated to a computer and overseen by a statistician (Krams, Lees et al. 2005, Wallace and Moodie 2014).

Both the doctor and the patient/subject undergo a discursive transformation in this evolution towards medicine as a decision analysis science. In spite of the discourse of tailored approaches to individual therapy, as Tutton notes, pharmacogenomics' promise (of shifting from universal therapy to greater specificity) is realized at the level of the sub-population, not the individual (Tutton 2012: 1726). In the testing process, individuals are mined for their information value, while only the most common, and therefore profitable, biomarker signatures are progressed through to development. As a result, holistic notions of the patient as a fully social as well as biological person are lost, with the potential for treatment inequalities to open up among rare, orphan, or scientifically disadvantaged populations. 
Hierarchical modeling and trial simulation exemplify Adams et al's (2009) notion of abduction, whereby the locus of reasoning moves temporally from the past to the future, using probabilistic anticipations to change the course of events in the present. Simulation, premised on the generation of virtual patients, has become a key feature of adaptive design trials. Writing fifteen years ago of the future of clinical trial simulation, Holford et al offered the following vision of the future of drug development:

In the far future, we foresee a central role for clinical trial simulation in a revolutionary paradigm shift in drug development practices. Today, drug development continues its inefficient tradition of many tens to hundreds of clinical trials per new drug application, supporting a mostly empirically derived safety-and-effectiveness database-with modest or no role of trial simulations. In our vision of a very different future, clinical trial simulations will be the principle [sic] scientific activity, and actual clinical trials will be few, aimed at informing simulation models and confirming simulation predictions. (Holford, Kimko et al. 2000: 231)

While simulation has not yet become the principal activity, it has nonetheless become a crucial underpinning to adaptive trials. Echoing Holford et al's vision, a panel discussion at the 2014 SMi conference on Adaptive Designs in Clinical Trials asked, 'How will trends toward modeled patients (physically and virtual) change the future of trials? Will we see trials without humans in our lifetime?' (SMi 2014).

Clinical trial simulation refers to the use of mathematical models to mimic the trial situation and conduct experiments which explore, describe, and predict the behaviour of that trial (Holford, Kimko et al. 2000, Giovagnoli and Zagoraiou 2012), with the aim of designing a trial under optimal assumptions. Thousands of virtual subjects can be simulated for any given trial, providing trial designers with different scenarios on accrual, 'dropout', and patient responses. Adaptive design trials, particularly those of a more complex nature, require simulation in a way which traditional trials do not. The more decisions or adaptations that are envisaged, the more difficult it is to know how the design of the trial will perform under different scenarios. Simulations allow trialists to assess the statistical properties of the design (e.g. type I error rate, power, sample size distribution), while varying inputs such as changing doses, different sample sizes, differing drug responses in subgroups, study 'dropout' rates and so on (for an example of trial simulation, see Maloney, Karlsson et al. 2007).

Proponents of adaptive design portray simulation as a powerful tool for overcoming uncertainty. We can recall that this was one of the original rationales for adaptive treatment allocation in the 1960s and 1970s, although at that time, uncertainty concerned the patient-clinician relationship in the context of alternative treatment choices. Following the discursive move to efficiency, uncertainty is recast as a property of the experiment per se, which can be mitigated through repeatedly simulating different trial scenarios. In the simulation discourse, the trial morphs from the humanistic to the mechanistic, as the following extract from an interview illustrates. Here, the trialist uses the analogy of car design to point to the logic of using simulation: 
It used to be that when you designed a car, what the factories actually did was they built every piece of the car and they drove the car, they ran it into walls, they tried stuff with it and then they changed it. Now, the way the car is a designed, $99 \%$ of it is done on a computer. All the pieces fit together, every little hole, every little screw. They model how strong everything is, they crash it into walls virtually, they drive it around tracks with curves virtually. And the first time they build that car, they have a very good idea of how it's going to perform and where the problems are going to be. OK. So we build airplanes on computers, we build cars on computers and we believe we can simulate them. And this is so much simpler than that.

A crucial difference, which this informant does not reference, is that cars and aeroplanes are made up of mechanical parts, whereas clinical trials are made up of people, whose behavioural complexity is of a different order completely. The freedom human beings have to change their minds, go in different directions, behave irrationally, and so on, is linked to uncertainty and indeterminacy in ways which this mechanistic view of the experiment does not acknowledge. In essence, the move to simulate patients empties them of their specificity, idiosyncracies and context, making them easily exchangeable, quantifiable and manipulable. The aim of simulation is not to represent what is, but to create what might be, and, through the application of mathematical know-how, build confidence in particular constellations of possibility.

A further criticism, made by one critic of adaptive designs who I spoke to, was that simulations are based on assumptions:

...you're simulating based on assumptions. And your assumptions may or may not be correct - and that's ridiculous. Simulations help; they help to model what the possibilities might be. They don't give you an answer.

And herein lies an important key to simulation, and to the current success of adaptive designs: the opening up of possibilities. As Adams et al observe, 'Management of the future becomes a pre-occupation of the present through the obligatory passage-point of "possibility." ...It is possible, so the injunction goes, to manage the anticipated" (Adams, Murphy et al. 2009: 259). Another way of putting this is to speak of the colonization of time, as does Barbara Adam (2004). While the rhetoric of simulation in adaptive designs saves time by disembedding it from social relations and thereby decontextualizing it, the propagation of the virtual extends the experimental landscape almost infinitely.

\section{Conclusion}

Hailed as the gold standard, the RCT occupies a hegemonic position at the top of Evidence Based Medicine's (EBM's) hierarchy of knowledge. In this paper, I asked how change is possible at the top of this hierarchy, and how adaptation is coming to replace standardization as a virtue. I sought to answer this through a genealogical approach, tracing arguments for and about adaptation in RCTs in the scientific literature from the 1950s to the present, and through interviews with key informants. I found that whereas adaptation was initially advocated for on the basis of patient wellbeing and the greater good, a marked change occurred in the 2000s, when the 
discourse shifted from ethics to efficiency. With the advent of highly sophisticated computer simulations, the focus has moved again to the merits of virtual trials and the savings these afford in moral and financial terms.

Key to understanding the rise in popularity of adaptive design is the changing use its advocates have made of the notion of experimental time. I have shown how the evolving discourses of time and patienthood have facilitated a move away from standardization as the singular logic of trials towards an appreciation of flexibility, undergirded by probabilistic methodologies. Adams et al's conceptual framework of anticipation has illuminated this evolving moral economy of medical research, in which modes of knowledge production which claim to know the future are supplanting the traditional certainties of fixed and standardized experimental designs. Predictable uncertainty, rather than promissory conjuration, is the currency of this emerging economy, which capitalizes on computer simulation and ever more sophisticated tools of prediction to leverage credibility.

Central to the possibility of change at the top of the EBM hierarchy, then, has been the combination of temporal expansionism in medical experimentation and better mathematical and computational means of quantifying, predicting and giving a sense of certainty to future unknowns. Against this backdrop, the scientific understanding of adaptation is shifting from a threat to the validity of the experiment to a means of controlling its outcome. So it is that advocates of adaptive design now speak of being able to fix experiments before they fail and being able to fail individual treatments faster. This renewed confidence in the clinical trial is turning the tables on the discourse of a broken system (DeVita 2008, Vickers 2014) and replacing it with a narrative of both future possibility and knowability. This is epitomized by marketing from Cytel, a clinical research organization specializing in adaptive trial design, which proclaims: 'When innovation is surer than convention' (Cytel no date). Predictability is recast, from an attribute of what has always been done, to an attribute of what it is possible to do through probabilistic methods.

While time and the management of uncertainty have become pillars of the paradigm shift within clinical research, the patient remains its raison d'être. The discursive transition from saving patients, to industry, to time itself has tracked the broader evolution in medicine from a holistic conception of the individual patient to a disembodied, virtual patient whose responses can be managed and understood computationally. While the commercial gains to be had from the colonization of time are by now self-evident, the rising popularity of adaptive design cannot be attributed to this alone. Indeed, as the increasing use of these methodologies in the public and not-for-profit sectors indicates, commercial value is but one facet of their appeal. It is at the intersection of temporality and patienthood that the moral, epistemic and commercial valences of adaptive design coalesce.

As Adams et al discuss, anticipation is 'a politics of temporality and affect' (Adams, Murphy et al. 2009: 246). Through the injunction to be constantly aware of and prepared for the future, anticipation leads to an ethicized state of being, in which the moral imperative is to be vigilant and act now in relation to the future. It is this normative dimension to the methodological developments of adaptive design, that, in combination with the changing temporality of the experimental landscape, has led to acceptance of change across the commercial and public sectors. In relation to the 
Ebola clinical trials, for example, the logic of adaptive design fits well with discourses of pandemic preparedness and global health securitization, which capitalize on the epidemiological rationalities of tracking, anticipating, and projecting to bring future events into the present (Thomas 2014). Likewise, in relation to cancer clinical trials, where the majority of methodological innovation has occurred, the moral and temporal politics of anticipation is a complimentary partner to the promissory narratives of molecularized cancer biologies (Rose 2007).

The move from standardization to adaptation, and the emerging moral economy of anticipation which makes it possible, have both theoretical and practical implications (of course, the two cannot entirely be separated). In practical terms, the temporal expansion of the experiment generates a number of new questions for clinical trialists, ethicists, and social scientists to consider: when does an experiment start and end? When is the experiment as originally conceived considered a different entity in the light of adaptations made? And how are success and failure being redefined by the ability to increase sample sizes, focus sampling on the populations most apt to respond, and add new arms as new treatments become available? To date, these questions have not been discussed in the literature. A debate about these issues needs to occur alongside the methodological elaboration of adaptive designs in statistical circles.

On top of such questions about the fundamental nature of the clinical trial are others about the standard of care. When experiment and provision of care become one and the same thing, the status of the patient is rendered ambiguous; it is no longer clear whether the priority is to treat the individual in their best interests or to extract maximum information value from them in the interests of the experiment. Such calculations will inevitably vary depending on who is asking the questions, with which goal in mind. As the Ebola clinical trials showed, the two are not always incompatible, but if commercial gain is driving the research, then efficiency rather than ethics is likely to have the upper hand.

To date, the implications of this for patients have been little considered. The analysis in this paper suggests that the logic of the experiment is no longer focused on a real and bounded patient population, but on a limitless set of data points, where the index of value is information rather than care. Linked to this, patients come to be thought of in terms of their probabilistic potential (through the use of virtual patients), rather than their actual needs and desires. These two facets push the debate about individual versus collective ethics heavily in the direction of the latter, where the gain is a potential acceleration in scientific answers to medical problems but the cost is a potential dehumanization of medical knowledge.

In terms of regulation, adaptation also gives rise to new questions. The traditional demarcation of trials into phases left space for regulators to assess knowledge gained before moving forwards. The abolition of this gap through the use of seamless designs, which is billed as saving time, means that such reflection time is instead replaced by the constant updating of a trial's success narrative. In addition, the timesaving implied by no longer having to make protocol amendments for changes which are anticipated in the protocol means another opportunity for regulatory intervention is bypassed. Rather than taking the claim to efficiency at face value, then, as critical researchers we need to ask in whose name and on which terms efficiency is enacted 
through the temporal politics of experimentation: efficiency for whom? Further empirical work might usefully explore what efficiency consists of as a deflated matter of practice, including which agencies and institutions are defining efficiency and how. At present, efficiency is a nebulous concept, undefined and with ambiguous parameters.

Finally, the theoretical implications of adaptation bear some consideration. To anticipate is literally to take into possession beforehand, from ante "before" and capere "to take". It is, as Adams et al write, "a moral economy in which the future sets the conditions of possibility for action in the present, in which the future is inhabited in the present" (249). This pre-empting of possibility is a form of temporal colonization, where action in the now is framed by imaginations and probabilistic calculations of what might be in the future. And yet, anticipation also leaves space for the unanticipated, the contrary, the improbable, the surprise (Adams, Murphy et al. 2009). The question is what happens when the unanticipated occurs.

In spite of all the talk of flexibility, adaptation here is highly formalized and regulated, with any potential changes to the design of experiments pre-specified, rulebased and centrally controlled. Changes are conceived in statistical rather than social terms, and reflect a preoccupation with what might be rather than what is on the ground. Just as STS teaches us that standardization is never fully achieved, so it seems likely that adaptive design would also be subject to the contingencies of the social world. How such real-time excesses are incorporated into the algorithmic approaches which drive adaptive trials remains to be seen. Can adaptively designed experiments embrace contextual diversity to become more situated, as social scientists have argued trials should? Or will they rather focus on ever more specific, prescribed, and therefore exclusionary experimental worlds in which patients are carefully selected to respond as predicted and local healthcare staff are divested of the power to make decisions?

When the goal is to fix the future as it unfolds, whose version of the future counts? The relationship of standardization to adaptation needs further theoretical elaboration on the basis of in-depth empirical work. Only then will we be able to see how the justificatory claims made for the moral economy of anticipation, described in the paper, radically alter or simply extend existing modes of control in the randomized controlled trial. 


\section{References}

Adam, B. (2004). Time. Cambridge, Polity Press.

Adams, V., M. Murphy and A. E. Clark (2009). "Anticipation: Technoscience, life, affect, temporality." Subjectivity 28: 246-265

Adebamowo, C., O. Bah-Sow, F. Binka, R. Bruzzone, A. Caplan, J.-F. Delfraissy, D. Heymann, P. Horby, P. Kaleebu, J.-J. M. Tamfum, P. Olliaro, P. Piot, A. Tejan-Cole, O. Tomori, A. Toure, E. Torreele and J. Whitehead (2014). "Randomised controlled trials for Ebola: practical and ethical issues." Lancet 384(9952): 1423-1424.

Anscombe, F. J. (1963). "Sequential medical trials." J Am Stat Assoc 58: 365-384. Armstrong, D. (2002). A New History of Identity. A Sociology of Medical Knowledge. Basingstoke, Palgrave.

Armstrong, D. (2012). "Screening: mapping medicine's temporal spaces." Sociology of Health \& Illness 34(2): 177-193.

Armstrong, D. (2014). "Chronic illness: a revisionist account." Sociology of Health \& Illness 36(1): 15-27.

Barker, A. D., C. C. Sigman, G. J. Kelloff, N. M. Hylton, D. A. Berry and L. J. Esserman (2009). "I-SPY 2: An Adaptive Breast Cancer Trial Design in the Setting of Neoadjuvant Chemotherapy." Clinical Pharmacology \& Therapeutics 86(1): 97-100.

Bartlett, R. H. (2014). "Clinical Research in Acute Fatal Illness: Lessons From Extracorporeal Membrane Oxygenation." Journal of Intensive Care Medicine. Bartlett, R. H., D. W. Roloff, R. G. Cornell, A. F. Andrews, P. W. Dillon and J. B. Zwischenberger (1985). "Extracorporeal circulation in neonatal respiratory failure: A prospective randomized study." Pediatrics 76(4): 479-487.

Berg, M. (1997). Rationalizing Medical Work: Decision Support Techniques and Medical Practices. Cambridge, MA, MIT Press.

Berry, D. (2006). "Bayesian Clinical Trials." Nature Reviews Drug Discovery: 27-36. Berry, S. M., E. A. Petzold, P. Dull, N. M. Thielman, C. K. Cunningham, G. R. Corey, M. T. McClain, D. L. Hoover, J. Russell, J. M. Griffiss and C. W. Woods (2016). "A response adaptive randomization platform trial for efficient evaluation of Ebola virus treatments: A model for pandemic response." Clinical Trials 13(1): 22-30. Bornkamp, B., F. Bretz, A. Dmitrienko, G. Enas, B. Gaydos, C. Hsu, F. Konig, M. Krams, Q. Liu, B. Neuenschwander, T. Parke, J. Pinheiro, A. Roy, R. Sax and F. Shen (2007). "Innovative approaches for designing and analyzing adaptive dose-ranging trials." J Biopharm Stat 17: 965-995.

Bosely, S. (2015) "Ebola: the race to find a cure." The Guardian.

Chuang-Stein, C., F. Bretz, O. Komiyama and J. Quinlan (2009). "Interactions With Regulatory Agencies to Enhance the Understanding and Acceptance of Adaptive Designs." Regulatory Focus 14(4): 36-42.

Colton, T. (1963). "A model for selecting one of two medical treatments." $\underline{\mathrm{J} \text { Am Stat }}$ Assoc 58: 388-401.

Cox, E., L. Borio and R. Temple (2014). "Evaluating Ebola Therapies - The Case for RCTs." New England Journal of Medicine 371(25): 2350-2351.

Cytel. (no date). "Cytel Adaptive Case Stduy 1." Retrieved 22/06/15, from http://www.cytel.com/hubfs/0-library-0/cases/case-1-medical-scienceadaptive-cardiovascular-trial.pdf.

Davis, C. and J. Abraham (2013). Unhealthy Pharmaceutical Regulation. Innovation, Politics and Promissory Science. Basingstoke, Palgrave Macmillan. DeVita, V. T. (2008). "The clinical trials system is broken." Nat Clin Prac Oncol 5(12): 683-683. 
Elliott, S. J. (1991). "Neonatal extracorporeal membrane oxygenation: how not to assess novel technologies." Lancet 337(8739): 476-478.

FDA (2004). Innovation or Stagnation? - Challenge and Opportunity on the Critical Path to New Medical Products. Silver Spring, U.S. Food and Drug Administration. FDA (2006). Critical Path Opportunities List. Silver Spring, U.S. Food and Drug Administration.

Fisher, J. A. (2009). Medical Research for Hire. The Political Economy of Pharmaceutical Clinical Trials New Brunswick, NJ and London, Rutgers University Press.

Freudenberg, N. (2014). Lethal But Legal: Corporations, Consumption, and Protecting Public Health. Oxford and New York, Oxford University Press. Gallo, P. and M. Krams (2006). "PhRMA Working Group on Adaptive Designs: Introduction to the Full White Paper." Drug Information Journal 40(4): 421-423. Giovagnoli, A. and M. Zagoraiou (2012). "Simulation of clinical trials: a review with emphasis on the design issues." Statistica LXXII(1): 63-80.

Hayden, E. C. (2014). "Ethical dilemma for Ebola trials." Nature 515: 177-178. Holford, N., S. C. Ma and B. A. Ploeger (2010). "Clinical Trial Simulation: A Review." Clinical Pharmacology \& Therapeutics 88(2): 166-182.

Holford, N. H. G., H. C. Kimko, J. P. R. Monteleone and C. C. Peck (2000). "Simulation of Clinical Trials." Annu. Rev. Pharmacol. Toxicol. 40: 209-234. Hutson, S. (2009). "A change is in the wind as 'adaptive' clinical trials catch on." Nat Med 15(9): 977.

Joffe, S. (2014). "Evaluating Novel Therapies During the Ebola Epidemic." JamaJournal of the American Medical Association 312(13): 1299-1300.

Jones, D. (2010). "Adaptive trials receive boost." Nat Rev Drug Discov 9(5): 345348.

Kanters, S., K. Thorlund and E. J. Mills (2015). "Ethical Testing of Experimental Ebola Treatments - Reply." Jama-Journal of the American Medical Association 313(4): 421-422.

Knox, R. A. (1989). A Harvard study on newborns draws lire: doctor faulted for limiting lifesaving therapy. Boston Globe: 25-27.

Krams, M., K. R. Lees and D. A. Berry (2005). "The Past Is the Future. Innovative Designs in Acute Stroke Therapy Trials." Stroke 36: 1341-1347.

Lang, T. (2011). "Adaptive Trial Design: Could We Use This Approach to Improve Clinical Trials in the Field of Global Health?" Am. J. Trop. Med. Hyg. 85(6): 967970.

Lantos, J. D. and J. Frader (1990). "Extracorporeal membrane oxygenation and the ethics of clinical research in pediatrics." N Engl J Med 323(6): 409-413.

Lewis, R., K. Viele, K. Broglio, S. Berry and A. Jones (2013). "An Adaptive, Phase II, Dose-finding Clinical Trial Design to Evaluate L-Carnitine in the Treatment Septic Shock Based on Efficacy and Predicted Probability of Subsequent Phase III Success." Critical Care Medicine 41(7): 1674-1678.

Lezaun, J. and C. M. Montgomery (2014). "The Pharmaceutical Commons: Sharing and Exclusion in Global Health Drug Development." Science, Technology \& Human Values.

Malcontent (1997). "Fumes from the spleen." Pediatric and Perinatal Epidemiology 11: $260-270$.

Maloney, A., M. O. Karlsson and U. S. H. Simonsson (2007). "Optimal adaptive design in clinical drug development: A simulation example." Journal of Clinical Pharmacology 47(10): 1231-1243. 
Miké, V., A. N. Krauss and S. Gail (1993). "Neonatal Extracorporeal Membrane Oxygenation (ECMO): Clinical Trials and the Ethics of Evidence." Journal of Medical Ethics 19(4): 212-218.

O'Rourke, P. P., R. K. Crone, J. P. Vacanti, J. H. Ware, C. W. Lillehei, R. B. Parad and M. F. Epstein (1989). "Extracorporeal membrane oxygenation and conventional medical therapy in neonates with persistent pulmonary hypertension of the newborn: a prospective randomized study." Pediatrics 84(6): 957-963.

Petryna, A. (2009). When Experiments Travel. Clinical Trials and the Global Search for Human Subjects. Princeton \& Oxford, Princeton University Press.

Petryna, A. (2009). When Experiments Travel. Clinical Trials and the Global Search for Human Subjects. Princeton \& Oxford, Princeton University Press.

Pocock, S. J. (1979). "Allocation of patients to treatment in clinical trials." Biometrics 35(1): 183-197.

Rose, N. (2001). "The Politics of Life Itself." Theory, Culture and Society 18(6): 130.

Rose, N. (2007). The Politics of Life Itself: Biomedicine, Power and Subjectivity in the Twenty-First Century. Princeton, NJ, Princeton University Press.

Salman, R. A., E. Beller, J. Kagan, E. Hemminki, R. S. Phillips, J. Savulescu, M.

Macleod, J. Wisely and Iain (2014). "Increasing value and reducing waste in

biomedical research regulation and management." Lancet 383: 176-185.

Simon, R. (1977). "Adaptive treatment assignment methods and clinical trials." Biometrics 33(4): 743-749.

SMi (2014). Adaptive Designs in CLinical Trials 24th \& 25th March 2014

Conference Information Pack.

Soll, R. F. (1996). "Neonatal extracorporeal membrane oxygenation-bridging technique." Lancet 348(9020): 70-71.

Sunder Rajan, K. (2006). Biocapital: The Constitution of Postgenomic Life. Durham, NC, Duke University Press.

Sunder Rajan, K. (2007). "Experimental Values: Indian Clinical Trials and Surplus

Health." New Left Review 45: 67-88.

Thomas, L. (2014). "Pandemics of the future: Disease surveillance in real time." Surveillance \& Society 12(2): 287-300.

Thompson, W. R. (1933). "On the likelihood that one unknown probability exceeds another in view of the evidence of two samples." Biometrika 25: 275-294.

Timmermanns, S. and M. Berg (2003). The gold standard. The challenge of evidencebased medicine and standardization in health care. Philadelphia, Temple University Press.

Timmermans, S. and M. Berg (1997). "Standardization in Action: Achieving Local Universality through Medical Protocols." Social Studies of Science 27(2): 273-305.

Tutton, R. (2012). "Personalizing medicine: Futures present and past." Social Science \& Medicine 75: 1721-1728.

Unguru, Y. (2011). "The successful integration of research and care: how pediatric oncology became the subspecialty in which research defines the standard of care." Pediatr Blood Cancer 56: 1019-1025.

Vickers, A. J. (2014). "Clinical trials in crisis: Four simple methodologic fixes." Clinical Trials.

Waldby, C. (2000). The Visible Human Project. London, Routledge.

Waldby, C. (2002). "Stem Cells, Tissue Cultures and the Production of Biovalue." Health: An Interdisciplinary Journal 6(3): 305-323. 
Wallace, M. P. and E. E. Moodie (2014). "Personalizing medicine: a review of adaptive treatment strategies." Pharmacoepidemiol Drug Saf. 23(6): 580-585.

Weinstein, M. C. (1974). "Allocation of Subjects in medical experiments." New England Journal of Medicine 291: 1278-1285.

Weinstein, M. C. (1974). "Allocation of subjects in medical experiments." N Engl J Med 291: 1278-1285.

Whitehead, J., P. Olliaro, T. Lang and P. Horby (2016). "Trial design for evaluating novel treatments during an outbreak of an infectious disease." Clinical Trials 13(1): 31-38.

Woodcock, J. and R. Woosley (2008). The FDA critical path initiative and its influence on new drug development. Annual Review of Medicine. 59: 1-12.

Zelen, M. (1969). "Play the Winner Rule and the Controlled Clinical Trial." Journal of the American Statistical Association 64(325): 131-146.

i For a complete overview of the designs and debates of the Ebola clinical trials, see the special issue 'Clinical Trials During the Ebola Crisis: A Series of Articles and Commentaries Describing the Challenges of Conducting Clinical Research During a Lethal Epidemic’ in Clinical Trials (2016) 13 (1).

ii In oncology, the integration of research and care is particularly notable, with clinical trials in the field of pediatric oncology, in particular, said to define the standard of care. The majority of children (approximately 70\%) suffering from cancer enroll in one or more clinical trials (Unguru 2011, Salman, Beller et al. 2014).

iii Based in Washington, PhRMA represents 56 biotechnology companies and "advocates in the U.S. and around the world on public policy issues critical to the discovery and development of innovative medicines" (http://www.phrma.org/about). For further analysis of the influence of PhRMA on Congress and on the FDA, see (Davis and Abraham 2013, Freudenberg 2014).

iv Search conducted in Nexis ${ }^{\circledR}$ on 17/03/15. Term 'adaptive design' searched in all 163 sources categorized as Industry = Pharmaceuticals and Biotechnology. 